\title{
Subthalamic nucleus stimulation in advanced Parkinson's disease: blinded assessments at one year follow up
}

\author{
B Ford, L Winfield, S L Pullman, S J Frucht, Y Du, P Greene, J H Cheringal, Q Yu, L J Cote, S Fahn, \\ G M McKhann II, R R Goodman
}

J Neurol Neurosurg Psychiatry 2004;75:1255-1259. doi: 10.1136/jnnp.2003.027557

See end of article for authors' affiliations

Correspondence to: Dr B Ford, Neurological Institute, 710 West 168th Street, New York, New York10032, USÁ; bford@ neuro.columbia.edu

Received 4September 2003 Revised 11 November 2003 Accepted 20 November 2003

\begin{abstract}
Objective: To measure the effect of deep brain stimulation (DBS) of the subthalamic nucleus in patients with advanced Parkinson's disease.

Design: Open label follow up using blinded ratings of videotaped neurological examinations.

Patients: 30 patients with advanced Parkinson's disease $(19$ male, 11 female; mean age 58.8 years; mean disease duration 12.8 years), complicated by intractable wearing off motor fluctuations and dopaminergic dyskinesias.

Main outcome measures: Unified Parkinson's disease rating scale (UPDRS), part III (motor), score at one year, from blinded reviews of videotaped neurological examinations. Secondary outcomes included the other UPDRS subscales, Hoehn and Yahr scale, activities of daily living (ADL) scale, mini-mental state examination (MMSE), estimates of motor fluctuations and dyskinesia severity, drug intake, and patient satisfaction questionnaire.

Results: Subthalamic nucleus stimulation was associated with a $29.5 \%$ reduction in motor scores at one year $(p<0.0001)$. The only important predictors of improvement in UPDRS part III motor scores were the baseline response to dopaminergic drugs $(p=0.015)$ and the presence of tremor $(p=0.027)$. Hoehn and Yahr scores and ADL scores in the "on" and "off" states did not change, nor did the mean MMSE score. Weight gain occurred in the year after surgery, from (mean) $75.8 \mathrm{~kg}$ to $78.5 \mathrm{~kg}(p=0.028)$. Duration of daily wearing off episodes was reduced by $69 \%$. Dyskinesia severity was reduced by $60 \%$. Drug requirements (in levodopa equivalents) declined by $30 \%$.

Conclusions: The 30\% improvement in UPDRS motor scores was a more modest result than previously reported. DBS did not improve functional capacity independent of drug use. Its chief benefits were reduction in wearing off duration and dyskinesia severity.
\end{abstract}

S ubthalamic nucleus (STN) stimulation is an effective treatment for advanced Parkinson's disease that remains levodopa responsive, but is complicated by wearing off motor fluctuations and dyskinesias. ${ }^{1-16}$ The many published series, describing three to 12 month outcomes in over 400 patients, have shown significant improvements in many aspects of Parkinson's disease, including tremor, bradykinesia, rigidity, dystonia, wearing off motor fluctuations, drug induced dyskinesias, and activities of daily living (ADL).

As experience with deep brain stimulation (DBS) accumulates, we have been impressed by the variation in outcome among patients who fit the generally accepted indication for DBS surgery, namely advanced Parkinson's disease complicated by wearing off motor fluctuations and dyskinesias. In our experience, while many patients derive an excellent result from surgery, others do not, for a variety of reasons that require further study. When patients with advanced Parkinson's disease have a good but not excellent response to levodopa, or later develop symptoms that do not respond to levodopa, the overall result of DBS may fall short of published expectations.

Much of the published surgical outcome data-consisting of ratings of ADL capacity, estimates of wearing off spells and dyskinesias, and patient diaries-are subject to placebo effects that can be robust and sustained for a year or more. ${ }^{17}$ Even among "blinded" assessments, the ratings have generally been done by study personnel familiar with the patients' symptoms and responses to DBS. ${ }^{18}$ However, for experienced clinicians, as well as patients, it is often possible to identify the stimulator status, ${ }^{3}{ }^{910}$ even when this is varied at random. Moreover, the unified Parkinson's disease rating scale (UPDRS) ${ }^{19}$ is a redundant scale ${ }^{20}$ which can multiply a clinical effect, especially when not administered in a completely blinded fashion.

One way to reduce bias is to present videotaped examinations in random order to an observer who is not part of the treating team. Our goal in the present study was to measure with optimal objectivity the clinical outcome of STN stimulation in a consecutive cohort of patients with advanced Parkinson's disease, using blinded ratings of videotaped neurological examinations.

\section{METHODS}

This study was carried out with the approval of Columbia University institutional review board under a Food and Drug Administration investigational device exemption. All subjects gave their informed consent for participation.

Patients underwent bilateral staged or simultaneous implantation of the Medtronic model 3387 or 3389 DBS lead with quadripolar stimulating electrode and Activa Itrel II (nine patients) or the Soletra (2l patients) multi-programmable, implantable pulse generator (IPG) (Medtronic, Minneapolis, Minnesota, USA). All operations were done by RRG, using a surgical technique described previously. ${ }^{521}$ The STN was targeted by combining direct magnetic resonance

Abbreviations: $A D L$, activities of daily living; $D B S$, deep brain stimulation; MMSE, mini-mental state examination; STN, subthalamic nucleus; UPDRS, unified Parkinson's disease rating scale 
imaging (MRI) with calculation relative to the intercommissural midpoint. Electrophysiological mapping was carried out using microelectrodes (FHC, Bowdoinham, Maine, USA) with an initial trajectory based on the MRI guidance calculation. An average of three parallel tracks was used to map STN boundaries and determine STN length, based on single unit firing patterns, macrostimulation, and responses to intraoperative clinical testing.

Patients were usually discharged from hospital on the second day after the brain surgery. The IPGs were implanted one week after the brain implants, as an outpatient procedure under general anaesthesia. After surgery, it was generally possible for patients to reduce their antiparkinsonian drug intake, although we did not use a formal tapering protocol to accomplish this. Patients returned to the study centre one week postoperatively, and then at six weeks, three months, six months, and one year. Deep brain stimulator settings were assessed and adjusted at each visit, when patients were in the unmedicated state.

For this analysis, patients were evaluated at baseline, one week before surgery, and at one year. Evaluations included the following: UPDRS, ${ }^{19}$ Hoehn and Yahr scale, ${ }^{22}$ Schwab and England ADL scale, ${ }^{23}$ mini-mental state examination (MMSE) scale, ${ }^{24}$ and a dyskinesia rating scale (see appendix 1). Selection criteria for the study were as follows:

- the presence of idiopathic Parkinson's disease, defined clinically by the presence of at least three of the four cardinal signs of the disease (tremor at rest, rigidity, bradykinesia, and postural instability);

- age of onset of Parkinson's disease over 29 years;

- the presence of disabling motor fluctuations or dyskinesias despite optimal medical management;

- absence of severe dementia (defined as an MMSE score $>22$ );

- the ability to give informed consent;

- acceptable general health.

Clinical assessments were carried out in the morning after the patients had been off their antiparkinsonian drugs overnight, using the core assessment program for intracerebral transplantation (CAPIT) protocol..$^{25}$ Patients underwent standardised videotaped neurological examinations in "on" and "off" states at baseline, and in three clinical states at one year: off medication/off stimulation, off medication/on stimulation, and on medication/on stimulation. The videotaped examinations were edited in random order onto VHS videocassettes for subsequent rating by one reviewer, who was blinded as to treatment status and was not part of the treatment team.

Additional data collected from patients included drug intake, weight, estimates of dyskinesia severity and amount of time spent in the off state each day, and estimates of overall treatment outcome and patient satisfaction (see appendix 2). We developed a new and simple dyskinesia rating scale that could be used by a videotape reviewer (appendix 1). Patients also underwent detailed neuropsychological testing, electrophysiological testing, and quality of life assessments, the results of which are not part of this report.

\section{Statistics}

Statistical analysis was done using SPSS version 10.0 software (SPSS Inc, Chicago, Illinois, USA). We conducted a series of comparisons between outcome measures using paired two sample $t$ tests for approximately normally distributed data, and non-parametric Wilcoxon signed-rank tests for discrete outcomes. Regression analyses were used to identify outcome predictors.

\section{RESULTS}

\section{General demographic and clinical data}

Between June 1999 and February 2001, 30 consecutive patients with advanced Parkinson's disease underwent bilateral STN electrode implantation. Eight patients underwent a staged procedure and 22 had a bilateral simultaneous implantation. The study included 19 men and 11 women (mean age at operation 58.8 years, range 36 to 80 ). The mean age of onset of Parkinson's disease was 47 years (range 29 to 72 ) and the mean disease duration was 12.8 years. Three patients had undergone bilateral putaminal human embryonic tissue graft implants, two a unilateral pallidotomy, and one a previous bilateral pallidotomy; these numbers were too small for separate analysis.

After one year of treatment, the stimulator settings were:

- Right electrodes $(\mathrm{n}=30): 24$ monopolar and six bipolar configuration; mean (SD) voltage amplitude, 3.0 (0.97) $\mathrm{mV}$ (range 1.5 to 5.9 ); mean pulse width $66.4 \mu \mathrm{s}$ (60 to 90); mean frequency $178.2 \mathrm{~Hz}$ (135 to 185 ).

- Left electrodes $(\mathrm{n}=30): 20$ monopolar and 10 bipolar configuration; mean (SD) voltage amplitude 2.7 (0.78) $\mathrm{mV}$ ( 1.5 to 4.3 ); mean pulse width $68.6 \mu$ s (60 to 90 ); mean frequency $178.6 \mathrm{~Hz}$ (135 to 185 ).

Two patients lived at a distance from the medical centre and were unable to return for their one year follow up evaluation; their clinical evaluation is not included in this report. Complete data were available on the remaining 28 patients.

\section{Principal outcome measures}

The primary end point for this study was a blinded evaluation of UPDRS part III motor scores using videotaped neurological examinations, comparing the one year result with baseline. In the unmedicated state, STN stimulation was associated with a $29.5 \%$ reduction in motor scores at one year $(p<0.0001)$ (range $-74 \%$ to $+20 \%$ ) (fig 1). The evaluation excluded rigidity scores, which cannot be viewed from a videotaped examination. However, when unblinded rigidity scores obtained at the time of the visit were added to the analysis, the result was statistically identical. Seven patients had no rest or action tremor at baseline, and these individuals experienced a $19.4 \%$ reduction in UPDRS part 3 motor scores, while those with tremor had a $37.4 \%$ decrease in motor scores $(p=0.027)$.

The cohort was improved overall but not all patients experienced individual benefit. Using a 30\% reduction in blinded UPDRS part III motor scores as a definition of individual success, only 12 of 28 patients $(42.8 \%)$ were greatly

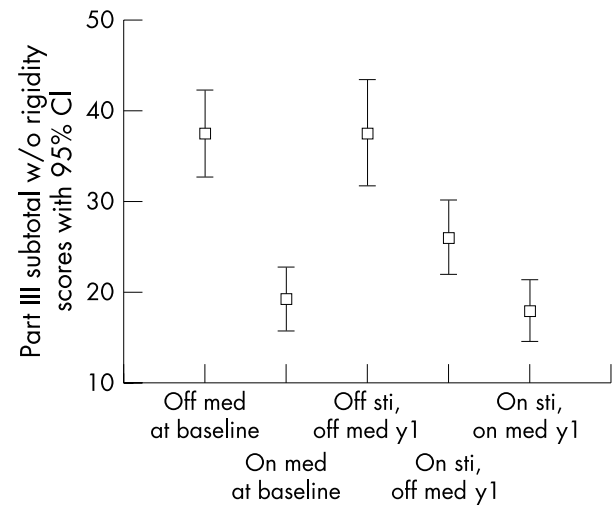

Figure 1 Blinded unified Parkinson's disease rating scale (UPDRS) part III motor scores (excluding rigidity) at baseline and one year. Cl, confidence interval; med, medication; sti, stimulation. 
improved by STN stimulation at the one year interval. Ten patients showed modest reductions in motor scores, ranging between $10 \%$ and $30 \%$, and six were unchanged. The 12 best responders to bilateral STN DBS, when analysed separately, showed an improvement of $52.5 \%$ in their UPDRS part III motor scores. From univariate regression analyses, the only important predictors of improvement in these scores were the baseline response to dopaminergic medication $(p=0.015)$ and the presence of tremor $(\mathrm{p}=0.027)$. Age at operation, duration of disease, age at disease onset, and baseline mental status scores were not predictive of outcome.

\section{Secondary outcome measures}

The outcome measures for the study are listed in table 1. UPDRS part 2 ADL subscores in the "off" state (off medication, on stimulation) improved by $6.8 \%$ over one year but this change was not statistically significant $(p=0.27)$. Patients' ADL scores in the "on" state worsened by $34.3 \%$ over one year, despite STN stimulation, a statistically significant change $(\mathrm{p}=0.031)$. When analysed individually, seven items contributed to this decline in "on" ADL capacity: speech, cutting food, dressing, hygiene, turning in bed, falling, and gait.

Comparing the one year score with the baseline score, the Hoehn and Yahr scores and the Schwab and England ADL scores in the "on" state (on medication, on stimulation) and in the "off" state (off medication, on stimulation) were not significantly changed as a result of DBS. The mean MMSE score (out of 30) was not significantly different after one year. Patients showed a significant weight gain during the year following surgery, increasing from a mean of $75.8 \mathrm{~kg}$ to $78.5 \mathrm{~kg}(\mathrm{p}=0.028)$.

At baseline, patients estimated their daily off time duration at 7.25 hours; at one year after surgery, the mean off time estimate was 2.25 hours $(\mathrm{p}<0.001)$. Two patients with severe off anxiety reported that this symptom resolved following DBS. Using a global dyskinesia rating scale (appendix 1), the mean dykinesia severity at baseline was 1.92. At one year, the dyskinesia severity was $0.76(\mathrm{p}<0.001)$.

Drug use declined and the interval between doses increased after deep brain stimulation (see table 2), although no formal medication reducing protocol was followed in this study. The daily levodopa intake was reduced from a mean of $1293 \mathrm{mg}$ at baseline to $1011 \mathrm{mg}$ at one year, a $22 \%$ decrease. The mean dopamine agonist intake decreased from 372 to
$221 \mathrm{mg} / \mathrm{d}$ (converted to levodopa equivalents) (table 2), a $41 \%$ reduction. Combining all dopaminergic drug treatment, the daily intake (in levodopa equivalents) declined from 1665 $\mathrm{mg}$ to $1160 \mathrm{mg}$, a $30 \%$ reduction. All these decreases in drug intake were statistically significant $(p<0.001)$. The mean interval between drug doses increased from 155 minutes at baseline to 191 minutes at one year $(p=0.081)$.

Using a global rating scale (appendix 2), patients and their families estimated the result of deep brain stimulation. The mean rating was 2 (range 0 to 3 ), suggesting that most patients believed their condition was moderately improved overall ("My condition is moderately improved but I still have some problems"). When asked if they were satisfied with the results of surgery, the mean response was 1.3 (range -1 to 3) ("I'm somewhat satisfied and happy with some improvements"), suggesting modest satisfaction overall.

\section{Complications}

In this series, nine serious complications-defined as potentially life threatening, or requiring surgical intervention or hospital admission-occurred in five patients. The complications included: ischaemic stroke (1), subdural haematoma (2), intracerebral haemorrhage (1), infection (3), chest wall haematoma (2). The three infections in this series occurred along the connecting wire, one requiring replacement of the DBS electrode, connector, and IPG. The ischaemic stroke and two chest wall haematomas occurred in one individual with factor XII deficiency who was considered a high risk candidate for these complications. Taken together, the incidence of infection requiring DBS lead replacement was $1.7 \%$ per electrode implant. The incidence of stroke or haemorrhage per electrode implant was $6.6 \%$. All patients

Table 2 Mean daily drug intake

\begin{tabular}{llll}
\hline Drug & Baseline & One year & $\%$ Change \\
\hline Levodopa & 1293 & 1011 & $-22 \%$ \\
Agonists & 372 & 221 & $-41 \%$ \\
COMT inhibitors & 214 & 145 & $-32 \%$ \\
Total levodopa equivalents* & 1665 & 1160 & $-30 \%$ \\
Interval between doses (min) & 155 & 191 & +0.23 \\
\hline
\end{tabular}

*Levodopa equivalents: $100 \mathrm{mg}$ levodopa is equivalent to: $10 \mathrm{mg}$ bromocriptine; $1 \mathrm{mg}$ pergolide; $1 \mathrm{mg}$ pramipexole; or $4 \mathrm{mg}$ ropinirole (standard and controlled release preparations are considered equal).

Table 1 Baseline and follow up variables

\begin{tabular}{lllll}
\hline & Baseline & 12 months & Change† & p Value \\
\hline Hoehn and Yahr score: off & 3.73 & 3.75 & $+0.5 \%$ & 0.98 \\
Hoehn and Yahr score: on & 2.65 & 2.52 & $-4.9 \%$ & 0.15 \\
Schwab and England ADL capacity: off & $37.0 \%$ & $41.8 \%$ & $+13.0 \%$ & 0.41 \\
Schwab and England ADL capacity: on & $76.4 \%$ & $75.2 \%$ & $-1.6 \%$ & 0.70 \\
UPDRS ADL (part 2): off & 26.4 & 24.6 & $-6.8 \%$ & 0.27 \\
UPDRS ADL (part 2): on* & 10.5 & 14.1 & $+34.3 \%$ & 0.031 \\
UPDRS motor (part 3): off med-off stim & 43.8 & 46.3 & $+5.7 \%$ & 0.26 \\
UPDRS motor (part 3): on med-on stim & 21.9 & 20.5 & $-6.4 \%$ & 0.39 \\
UPDRS motor (part 3): off med-on stim & - & 32.3 & - & - \\
UPDRS (total): off & 70.7 & 70.9 & $+0.3 \%$ & 0.94 \\
UPDRS (total): on* & 32.0 & 34.0 & $+6.1 \%$ & 0.44 \\
MMSE score (out of 30) & 28.1 & 26.6 & -1.5 & 0.052 \\
Drug intake (l-dopa equivalents) & 1665 & 1160 & -505 & $<0.001$ \\
Duration of daily off time (hours) & 7.25 & 2.25 & $-69 \%$ & $<0.001$ \\
Dyskinesia severitył & 1.92 & 0.76 & -60.4 & $<0.001$ \\
Weight (kg) & 75.8 & 78.5 & +2.8 & 0.028 \\
\hline
\end{tabular}

*At 12 months, the "on" state reflects on medication and on stimulation.

tFor Hoehn and Yahr scores and UPDRS scores, a minus sign indicates improvement and a plus sign indicates deterioration.

fDyskinesia rating scale is described in appendix 1 .

med, medication; MMSE, mini-mental state examination; stim, stimulation; UPDRS, unified Parkinson's disease rating scale. 
experiencing these events in our series had minor or short lived (less than 72 hours) neurological symptoms, and made a complete recovery. Two patients with mild pre-existing cognitive impairment (MMSE 26/30) experienced confusion that lasted several weeks postoperatively, but this resolved. In addition to complications caused by the operation or hardware, almost every patient experienced adverse reversible effects of stimulation, including unpleasant tingling sensations, muscular contractions, imbalance or ataxia, speech slurring, and unusual cephalic sensations, as previously reported.

\section{DISCUSSION}

This series provides an objective measure of the clinical effects of STN stimulation in patients with advanced Parkinson's disease complicated by wearing off motor fluctuations and dopaminergic dyskinesias. Using blinded ratings of videotaped neurological examinations, we found an approximately $30 \%$ improvement in UPDRS motor scores attributed to the effect of DBS alone, comparing the off medication-on stimulation state at one year with the unmedicated state at baseline. Moreover, a subgroup of the best responders to DBS, comprising $40 \%$ of the entire cohort, achieved a reduction in motor scores of $52.5 \%$. Patients with tremor showed the greatest improvement in UPDRS motor scores following DBS, even when the tremor items were removed from the analysis. A higher prevalence of tremor patients is likely to influence the overall outcome of DBS studies.

In contrast to other reports, we found that STN stimulation alone was inferior to drug treatment in its antiparkinsonian effect. The effect of STN stimulation, as can be seen in fig l, was intermediate between the off medication state and the on medication state. Despite changes in motor scores, in contrast to other studies patients in this series did not experience benefit in their capacity for daily living or in their Hoehn and Yahr stage, comparing off and on scores at baseline and follow up. The critical determinants of Hoehn and Yahr staging are postural stability and falling, variables that did not improve as a result of DBS. The chief benefits of DBS in our series were reduced duration of "off" spells and reduced duration and severity of dyskinesias.

Following successful STN stimulation, many patients can reduce their drug treatment, although this varies between patients and institutions. At some institutions, medication reduction is an explicit goal of surgery, and patients follow a protocol of weaning that begins at the time of surgery. ${ }^{73}{ }^{15}$ In our series, we found that most patients could not tolerate being off their drug treatment postoperatively, despite effective stimulation. An important contributor to the reduced medication intake was an increase in the dose intervals, and not a reduction in individual doses. While the duration of wearing off spells was prominently shortened, patients continued to experience fluctuations. The severity of "off" spells, as measured during the off medication-on stimulation state, was approximately one third less severe overall.

In this report, and most published analyses to date, the most important predictor of surgical outcome was the effect of levodopa on a patient's baseline parkinsonism. Some have advocated the use of an apomorphine test ${ }^{26}$ or a suprathreshold dose of levodopa ${ }^{7}$ to aid the selection criteria for prospective patients. The reduction in UPDRS motor scores at baseline, comparing "on" and "off" states, in most published series ranges from $45 \%{ }^{3}{ }^{14}$ to about $72 \%,{ }^{5}{ }^{16}$ and in the present series it was $50 \%$. In general, our results were more modest than those reported elsewhere but we believe they represent a more realistic estimate of the effect of DBS on Parkinson's disease. We do not discount the possibility that our results may reflect differences in patient selection or surgical technique.
The present series is small and the duration of follow up is short relative to the time course of the disease after surgery. Long term studies, with complete follow up, are necessary to determine the larger impact of DBS on Parkinson's disease. To date, one half of the published reports provide less than six months' follow up, including the largest series to date. ${ }^{1}$ In many patients, levodopa responsiveness declines as the disease progresses. For these individuals, it can be anticipated that the result of DBS may also decline over time. In addition, many patients-including participants in this series-develop symptoms that are unresponsive to drug treatment. One study documented a decline in UPDRS motor scores after the first year, with a loss of benefit in axial subscores by the 24 month end point. ${ }^{27}$ The five year follow up study of the Grenoble cohort showed persisting benefits in tremor and rigidity but declining effects on akinesia, speech, gait freezing, and postural stability, ${ }^{28}$ findings observed in the present study.

There is an emerging consensus that the ideal patients for DBS are young and have tremors, have an excellent response to levodopa, and experience severe dyskinesias. ${ }^{16}$ Unfortunately, many patients with advanced Parkinson's disease do not fit this clinical profile. In our experience, many individuals with intractable motor fluctuations and dyskinesias also have minor cognitive impairment, postural instability, and levodopa refractory symptoms, such as freezing, tachyphemia, and poor posture. This sizable subgroup of patients, in our opinion, derives less than complete benefit from STN stimulation and thus presents a therapeutic challenge for which further refinements in devices and techniques are clearly needed.

\section{ACKNOWLEDGEMENTS}

This work was supported by the Parkinson's Disease Foundation, New York, and the Kroll-Schreiner Fund.

\section{Authors' affiliations}

B Ford, L Winfield, S L Pullman, Q Yu, G M McKhann, R R Goodman, Center for Movement Disorders Surgery, Columbia-Presbyterian Medical Center, Columbia University, New York, USA

S J Frucht, P Greene, J H Cheringal, L J Cote, S Fahn, Department of Neurology, Columbia-Presbyterian Medical Center

Y Du, Center for Biostatistics and Epidemiology, Columbia-Presbyterian Medical Center

Competing interests: none declared

\section{APPENDIX 1}

\begin{tabular}{ll}
\hline Dyskinesia rating scales & \\
\hline Videotape reviewer's scale & Patient's self rating scale \\
\hline $\begin{array}{l}\text { No dyskinesias } \\
1 \text { Mild dyskinesias }\end{array}$ & 0 No dyskinesias \\
2 Obvious, moderately severe & 1 Mild and hardly noticeable \\
$\begin{array}{l}\text { dyskinesias that would not } \\
\text { necessarily merit a change in } \\
\begin{array}{l}\text { antiparkinsonian medication } \\
\text { Severe, ballistic dyskinesias } \\
\text { that would require intervention }\end{array}\end{array}$ & $\begin{array}{l}\text { live with the problem } \\
\text { Severe and disabling dyskinesias }\end{array}$ \\
\hline
\end{tabular}

\section{APPENDIX 2}

\section{GLOBAL RATING SCALE - PATIENT'S SELF ASSESSMENT QUESTIONNAIRE}

I. What is the effect of deep brain stimulation on your condition?

3. My condition is greatly improved-almost to normal.

2. My condition is moderately improved-but I still have some problems. 
1. My condition is minimally improved-my problems are almost the same as before.

0 . My condition is no better or the same as it was before the surgery.

-1 . My condition is a little worse than before the surgery.

-2 . My condition is worse than before the surgery.

-3 . My condition is much worse than before the surgery.

\section{Are you satisfied with the results of deep brain} stimulation on your condition?

3. Completely satisfied. The surgery has given me a normal life again.

2. Very satisfied. I'm doing well and my problems are much less than before.

1. Somewhat satisfied. I'm happy with some improvements.

0 . I'm not unhappy with the results but my problems are basically the same.

-1 . I'm a little disappointed in the results. I might not have gone through the surgery if I'd known.

-2 . I'm very disappointed. It was not worth all the trouble I went through.

-3. Surgery made my condition worse.

\section{REFERENCES}

1 Deep-Brain Stimulation for Parkinson's Disease Study Group. Deep-brain stimulation of the subthalamic nucleus or the pars interna of the globus pallidus in Parkinson's disease. N Engl J Med 2001;345:956-63.

2 Krack P, Pollak P, Limousin P, et al. Stimulation of subthalamic nucleus alleviates tremor on Parkinson's disease. Lancet 1998;350:1675.

3 Kumar R, Lozano AM, Kim YJ, et al. Double-blind evaluation of subthalamic nucleus deep brain stimulation in advanced Parkinson's disease. Neurology 1998:51:850-5.

4 Limousin P, Pollak P, Benazzouz A, et al. Bilateral subthalamic nucleus stimulation for severe Parkinson's disease. Mov Disord 1995; 10:672-4

5 Limousin P, Krack P, Pollak P, et al. Electrical stimulation of the subthalamic nucleus in advanced Parkinson's disease. N Engl J Med 1998:339:1 105-11.

6 Lopiano L, Rizzone M, Bergamasco B, et al. Deep brain stimulation of the subthalamic nucleus: clinical effectiveness and safety. Neurology 2001;56:552-4

7 Molinuevo JL, Valldeoriola F, Tolosa E, et al. Levodopa withdrawal after bilateral subthalamic nucleus stimulation in advanced Parkinson disease. Arch Neurol 2000;57:983-8

8 Ostergaard K, Sunde N, Dupont E. Effects of bilateral stimulation of the subthalamic nucleus in patients with severe Parkinson's disease and motor fluctuations. Move Disord 2002;17:693-700.

9 Pinter MM, Alesch F, Murg M, et al. Deep brain stimulation of the subthalamic nucleus for control of extrapyramidal symptoms in advanced idiopathic
Parkinson's disease: one year follow-up. J Neural Transm 1999; 106:693-709.

10 Simuni T, Jaggi JL, Mulholland H, et al. Bilateral stimulation of the subthalamic nucleus in patients with Parkinson's disease: a study of efficacy and safety. J Neurosurg 2002;96:666-72.

11 Tavella, Bergamasco B, Bosticco E, et al. Deep brain stimulation of the subthalamic nucleus in Parkinson's disease: long-term follow-up. Neurol Sci 2002;23:S21 1-12.

12 Thobois S, Mertens $P$, Guenot $M$, et al. Subthalammic nucleus stimulation in Parkinson's disease. J Neurol 2002;249:529-34.

13 Valldeoriola $F$, Pilleri $M$, Tolosa $E$, et al. Bilateral subthalamic stimulation monotherapy in advanced Parkinson's disease: long-term follow-up of patients. Move Disord 2002:17:125-32.

14 Vesper J, Klosterman F, Stockhammer F, et al. Results of chronic subthalamic nucleus stimulation for Parkinson's disease: a 1-year follow-up study. Surg Neurol 2002;57:306313.

15 Vingerhoets FJG, Villemure J-G, Temperli P, et al. Subthalamic DBS replaces levodopa in Parkinson's disease. Neurology 2002;58:396-401.

16 Welter ML, Houeto JL, Tezanas du Montcel S, et al. Clinical predictive factors of subthalamic nucleus stimulation in Parkinson's disease. Brain 2002; 125:575-83.

17 Freed C, Greene PE, Breeze RE, et al. Transplantation of embryonic dopamine neurons for severe Parkinson's disease. N Engl J Med 2001;344:710-19.

18 Ford B. Deep-brain stimulation in Parkinson's disease. N Engl J Med 2002;346:452-3.

19 Fahn S, Elton RL. The unified Parkinson's disease rating scale. In: Fahn S Marsden CD, Calne DB, et al, eds. Recent developments in Parkinson's disease, vol 2. Florham Park, NJ: MacMillan Healthcare Information, 1987:293-304

20 Martignoni E, Franchignoni F, Pasetti C, et al. Psychometric properties of the Unified Parkinson's Disease Rating Scale and of the Short Parkinson's Evaluation Scale. Neurol Sci 2003;24:190-1.

21 Starr PA, Christine CW, Theodosopoulos PV, et al. Implantation of deep brain stimulators into the subthalamic nucleus: technical approach and magnetic resonance imaging-verified lead locations. J Neurosurg 2002;97:370-87.

22 Hoehn MM, Yahr MD. Parkinsonism: onset, progression and mortality. Neurology 1967; 17:427-2.

23 Schwab RS, England AC. Projection technique for evaluating surgery in Parkinson's disease. In: Gillingham FJ, Donaldson IML, eds. Third Symposium on Parkinson's Disease. Edinburgh: Churchill Livingstone, 1969:152-7.

24 Folstein MF, Folstein SE, McHugh PR. "Mini-mental state" - a practica method for grading the mental status of patients for the clinician. J Psychiatr Res 1975;12:189.

25 Langston JW, Widner H, Goetz CG. Core assessment program for intracerebral transplantations (CAPIT). Mov Disord 1992;7:2-13.

26 Varma TRK, Fox SH, Eldridge PR, et al. Deep brain stimulation of the subthalamic nucleus: effectiveness in advanced Parkinson's disease patients previously reliant on apomorphine. J Neurol Neurosurg Psychiatr 2003;74:170-4.

27 Kleiner-Fisman G, Fisman DN, et al. Long-term follow-up of bilateral subthalamic nucleus deep brain stimulation in patients with advanced Parkinson's disease: the Toronto Western Hospital experience. J Neurosurg 2003;99:489-95

28 Krack P, Batir A, Van Blercom N, et al. Five year follow-up of bilateral stimulation of the subthalamic nucleus in advanced Parkinson's disease. N Engl J Med 2003;349:1925-34. 